\title{
Mycobacterium tuberculosis Beijing Lineage and Risk for Tuberculosis in Child Household Contacts, Peru
}

\author{
Chuan-Chin Huang, ${ }^{1}$ Alexander L. Chu, ${ }^{1}$ Mercedes C. Becerra, Jerome T. Galea, \\ Roger Calderón, Carmen Contreras, Rosa Yataco, Zibiao Zhang, Leonid Lecca, Megan B. Murray
}

Few studies have prospectively compared the relative transmissibility and propensity to cause disease of $M y$ cobacterium tuberculosis Beijing strains with other human-adapted strains of the M. tuberculosis complex. We assessed the effect of Beijing strains on the risk for $M$. tuberculosis infection and disease progression in 9,151 household contacts of 2,223 culture-positive pulmonary tuberculosis (TB) patients in Lima, Peru. Child contacts exposed to Beijing strains were more likely than child contacts exposed to non-Beijing strains to be infected at baseline, by 12 months of follow-up, and during followup. We noted an increased but nonsignificant tendency for child contacts to develop TB. Beijing strains were not associated with TB in adult contacts. These findings suggest that Beijing strains are more transmissible in children than are non-Beijing strains.

$\mathrm{T}$ uberculosis (TB) remains the leading cause of 1 death worldwide from a single infectious disease; in $2017, \approx 10$ million incident cases and 1.7 million deaths were reported (1). The causative pathogen, Mycobacterium tuberculosis, is divided into 7 humanadapted phylogenetic lineages, of which some are geographically restricted and others are widespread throughout the world, possibly because they are better adapted to environments of high human density (2). One of the widespread groups, lineage 2, includes a widely distributed genotype, the Beijing strain, that has been repeatedly implicated in outbreaks and in the evolution of drug resistance (3-6). The global

\footnotetext{
Author affiliations: Brigham and Women's Hospital, Boston, Massachusetts, USA (C.-C. Huang, M.C. Becerra, Z. Zhang, M.B. Murray); Harvard Medical School, Boston (C.-C. Huang, M.C. Becerra, M.B. Murray); Harvard University Division of Continuing Education, Cambridge, Massachusetts, USA (A.L. Chu); University of South Florida, Tampa, Florida, USA (J.T. Galea); Socios En Salud Sucursal, Lima, Peru (R. Calderón, C. Contreras, R. Yataco, L. Lecca)
}

distribution of Beijing strains and their precipitous rise in some populations have led researchers to speculate that it may be more transmissible and more likely to cause disease than other less widely distributed M. tuberculosis lineages (7-9).

However, the few direct assessments of the relative transmissibility of Beijing strains have been inconclusive. Although some studies found that exposure to Beijing strains was more likely than exposure to other strains to lead to TB (10-15), others reported no difference $(16,17)$. Several studies have suggested that the Beijing genotype is more common among young persons and that its frequency declines with age (18-21). To explore these factors, we directly compared the relative transmissibility and propensity to cause disease of Beijing strains with other strains in a cohort study of household contacts of patients with pulmonary TB in Lima, Peru.

\section{Methods}

\section{Ethics Statement}

The Institutional Review Board of Harvard School of Public Health and the Research Ethics Committee of the National Institute of Health of Peru approved the study. All study participants or their guardians provided written informed consent, and children $<18$ years of age provided assent.

\section{Setting, Study Design, and Participant Recruitment and Follow-Up}

The study design and methods were previously described in detail (22). In brief, we conducted a prospective cohort study of household contacts of pulmonary TB patients in Lima, Peru. The study area comprised 20 districts inhabited by $\approx 3.3$ million residents living in urban areas and in periurban, informal shantytown settlements.

\footnotetext{
${ }^{1}$ These authors contributed equally to this article.
} 
During September 2009-August 2012, we identified patients $\geq 15$ years of age who had received a diagnosis of clinically presumptive pulmonary TB at any of 106 participating health centers. We confirmed the microbiological status of their disease with either a positive sputum smear or mycobacterial culture. We also recorded the index patient's sociodemographic data; baseline smear status; presence or absence of cavitary disease; tobacco and alcohol use; HIV status; time from symptom onset until initiation of treatment; and, for patients, with drug-resistant TB, the time from TB diagnosis until start of an effective treatment (i.e., a drug regimen deemed appropriate for the index patient's drug-resistant profile).

Within 2 weeks after index patient diagnosis, we enrolled all consenting household contacts. We assessed baseline $M$. tuberculosis infection status with the tuberculin skin test (TST) in household contacts with no history of a positive TST or TB. Household contacts who had signs or symptoms of TB underwent clinical evaluation and, if indicated, initiated treatment under Peru's National Tuberculosis Program guidelines (23). We offered HIV testing to all study participants. In accordance with Peru's National Tuberculosis Program guidelines, isoniazid preventive therapy (IPT) was offered to household contacts $\leq 19$ years of age and persons with specified concurrent conditions. At the time of household contact enrollment, we collected age, sex, sociodemographic, and occupational information; height and weight; alcohol and tobacco use information; HIV status; self-reported diabetes mellitus; history and presence of $M$. bovis BCG vaccination scars; use of IPT; history of TB; and housing features. We repeated the TST 6 and 12 months after initial evaluation among household contacts who had previously tested negative; 2, 6, and 12 months after initial evaluation, we reevaluated them for pulmonary and extrapulmonary TB. We also accessed the medical records of health centers in the catchment areas to identify household contacts whose TB was diagnosed at a health center during the follow-up period.

\section{Whole-Genome Sequencing}

Among 3,027 index patients with culture-positive isolates at baseline, 2,143 had isolates that also underwent whole-genome sequencing (WGS) using the Illumina HiSeq 4000 platform (Illumina, https:/ / www.illumina.com) with a read length of 100-150 bp and $\geq 50$-fold coverage. The raw sequence data were trimmed using the sickle package (24) and mapped to the H37Rv reference genome using the BWA-MEM algorithm (25). We used a coverage-based approach,
Table 1. Baseline characteristics of Mycobacterium tuberculosis culture-positive index patients, Lima, Peru, September 2009_ August 2012

\begin{tabular}{|c|c|}
\hline Variable & No. $(\%), \mathrm{N}=2,223^{*}$ \\
\hline \multicolumn{2}{|l|}{ Age, y } \\
\hline $16-30$ & $1,363(61)$ \\
\hline $31-45$ & $465(21)$ \\
\hline$\geq 46$ & $395(18)$ \\
\hline \multicolumn{2}{|l|}{ Sex } \\
\hline M & $1,289(58)$ \\
\hline $\mathrm{F}$ & $934(42)$ \\
\hline \multicolumn{2}{|l|}{ Concurrent condition } \\
\hline HIV seropositive & $59(3)$ \\
\hline Self-reported diabetes & $111(6)$ \\
\hline Current smoker & $60(3)^{\prime}$ \\
\hline \multicolumn{2}{|l|}{ M. tuberculosis lineage } \\
\hline L2 (Beijing) & $255(12)$ \\
\hline L4.1 & $951(43)$ \\
\hline L4.3 & 775 (35) \\
\hline Other & $242(11)$ \\
\hline \multicolumn{2}{|l|}{ Sputum smear status $†$} \\
\hline Negative & $548(25)$ \\
\hline+ & $639(29)$ \\
\hline++ & $431(19)$ \\
\hline+++ & $596(27)$ \\
\hline Cavitary disease & $655(30)$ \\
\hline \multicolumn{2}{|l|}{ Drug resistance profile } \\
\hline Pansusceptible & $1,442(67)$ \\
\hline Resistant & $726(33)$ \\
\hline
\end{tabular}

specifically SAMtools (default settings) (26) and pilon (27), to identify single-nucleotide polymorphisms across the whole genome. We assigned a call as missing if the valid depth of coverage at a specific site was $<8$ reads, if the mean read-mapping quality at the site did not reach 9 , or if none of the alternative alleles accounted for $\geq 85 \%$ of the valid coverage.

\section{Lineage Identification of $\boldsymbol{M}$. tuberculosis Strains}

Among index patients for whom WGS data were available, we identified $M$. tuberculosis lineages and sublineages on the basis of a previously published barcode that differentiates the groups on the basis of singlenucleotide polymorphisms (28). For index patients without available WGS data, we genotyped their $M$. tuberculosis isolates using 24-locus mycobacterial interspersed repetitive units-variable-number tandem repeats (MIRU-VNTR) (29). We used the best-match algorithm from the MIRU-VNTRplus online database (http://www.miru-vntrplus.org) to identify the lineages of the typed samples by cross-referencing them with reference lineages stored on the database (30).

\section{Outcomes}

We considered 4 distinct outcomes: $M$. tuberculosis infection at baseline, time to TST conversion during the 12 months of follow-up, M. tuberculosis infection by 
Table 2. Baseline characteristics of household contacts exposed to a Mycobacterium tuberculosis culture-positive index tuberculosis patient, Lima, Peru, September 2009-August 2012

\begin{tabular}{|c|c|c|c|}
\hline Variable & Total no. (\%), $\mathrm{N}=9,151^{*}$ & Age $\leq 15$ y, no. (\%) & Age >15 y, no. (\%) \\
\hline $\begin{array}{r}\text { Age, } y \\
\leq 15 \\
>15 \\
\end{array}$ & $\begin{array}{l}3,225(35) \\
5,926(65)\end{array}$ & $\begin{array}{l}3,225(100) \\
\text { Not applicable }\end{array}$ & $\begin{array}{l}\text { Not applicable } \\
5,926(100)\end{array}$ \\
\hline $\begin{array}{l}\text { Sex } \\
\text { M } \\
\mathrm{F} \\
\end{array}$ & $\begin{array}{l}4,147(45) \\
5,004(55)\end{array}$ & $\begin{array}{l}1,620(50) \\
1,605(50)\end{array}$ & $\begin{array}{l}2,527(43) \\
3,399(53)\end{array}$ \\
\hline $\begin{array}{l}\text { Concurrent condition } \\
\text { HIV seropositive } \\
\text { Self-reported diabetes } \\
\text { Current smoker }\end{array}$ & $\begin{array}{l}33(<1) \\
165(2) \\
559(6)\end{array}$ & $\begin{array}{l}3(<1) \\
2(<1) \\
4(<1)\end{array}$ & $\begin{array}{c}30(1) \\
163(3) \\
555(10)\end{array}$ \\
\hline $\begin{array}{l}\text { M. tuberculosis lineage exposure } \\
\text { L2 (Beijing) } \\
\text { L4.1 } \\
\text { L4.3 } \\
\text { Other } \\
\text { Presence of M bovis BCG vaccination scar }\end{array}$ & $\begin{array}{c}1,041(11) \\
3,733(41) \\
3,416(37) \\
961(11) \\
8,110(89)\end{array}$ & $\begin{array}{c}349(11) \\
1,332(41) \\
1,202(37) \\
342(11) \\
2876(89)\end{array}$ & $\begin{array}{c}692(12) \\
2,401(41) \\
2,214(37) \\
619(10) \\
5253(89)\end{array}$ \\
\hline $\begin{array}{l}\text { Nutritional status } \\
\text { Normal weight } \\
\text { Underweight } \\
\text { Overweight }\end{array}$ & $\begin{array}{c}5,261(58) \\
163(2) \\
3,636(40)\end{array}$ & $\begin{array}{c}2,527(79) \\
95(3) \\
565(18)\end{array}$ & $\begin{array}{c}2,734(47) \\
68(1) \\
3,071(52)\end{array}$ \\
\hline $\begin{array}{l}\text { Socioeconomic status } \\
\text { Low } \\
\text { Middle } \\
\text { High } \\
\end{array}$ & $\begin{array}{l}3,112(35) \\
3,991(45) \\
1,828(21)\end{array}$ & $\begin{array}{c}1,253(40) \\
1,384(44) \\
514(16)\end{array}$ & $\begin{array}{l}1,859(33) \\
2,607(45) \\
1,314(23)\end{array}$ \\
\hline $\begin{array}{l}\text { Isoniazid preventive therapy recipient } \\
\text { "Numbers might not add to total because of missin }\end{array}$ & $2,090(23)$ & $1,542(48)$ & $490(7)$ \\
\hline
\end{tabular}

12 months of follow-up, and incident disease during the 12-month follow-up time. We classified household contacts as infected at baseline if they reported a history of TB, reported a previous positive TST result within 6 months after study enrollment, or had a positive TST (induration size $\geq 10 \mathrm{~mm} \mathrm{[} \geq 5 \mathrm{~mm}$ for HIV-positive household contacts]) at baseline. We considered initially TST-negative household contacts to have undergone TST conversion if their TST status converted from negative to positive or if TB developed during follow-up. We considered household contacts to be infected with $M$. tuberculosis by 12 months of follow-up if contacts were infected at baseline or had a TST conversion during the 12-month follow-up period (22). We considered household contacts to have co-prevalent TB if it was diagnosed within 2 weeks after the diagnosis in the index patient. If household contacts received diagnoses 2 weeks-15 months (1year follow-up plus a 3-month buffer period) after index patient diagnosis, we considered incident TB to have developed in those contacts during follow-up. We based TB diagnosis among contacts $\geq 18$ years of age on the same criteria we used for index patients; the diagnosis among household contacts $<18$ years was based on published consensus guidelines from an expert panel on classifying TB in children (31).

\section{Statistical Analysis}

We made an a priori decision to analyze child household contacts ( $\leq 15$ years) and adult household contacts (>15 years) separately for several reasons. First, we considered child contacts to be a more representative age group for household-based TB transmission than their more mobile, socially active adult counterparts. Second, several previous studies suggest that Beijing strains more commonly affect children than adults (18-20). We used complete data analyses in all multivariate adjustment models and conducted all statistical analyses in $\mathrm{R}$ version 3.5.1 (https:// www.r-project.org).

Table 3. Effect of the Mycobacterium tuberculosis Beijing lineage on the risk for infection at baseline in child and adult household contacts of culture-positive index patients, Lima, Peru, September 2009-August 2012

\begin{tabular}{|c|c|c|c|c|c|c|}
\hline \multirow[b]{3}{*}{ Lineage } & \multicolumn{3}{|c|}{ Age $\leq 15$ y, $n=3,115^{*}$} & \multicolumn{3}{|c|}{ Age $>15 y, n=5,663^{*}$} \\
\hline & \multirow{2}{*}{$\begin{array}{c}\text { Prevalence, } \\
\text { no. }(\%) \dagger\end{array}$} & \multicolumn{2}{|c|}{ Risk ratio $(95 \% \mathrm{Cl})$} & \multirow{2}{*}{$\begin{array}{c}\text { Prevalence, } \\
\text { no. }(\%) \dagger\end{array}$} & \multicolumn{2}{|c|}{ Risk ratio $(95 \% \mathrm{Cl})$} \\
\hline & & Univariate & Multivariate $\ddagger$ & & Univariate & Multivariatef \\
\hline Non-Beijing & $731(26)$ & Referent & Referent & $2,654(53)$ & Referent & Referent \\
\hline Beijing & $110(33)$ & $1.27(1.06-1.53)$ & $1.23(1.02-1.50)$ & $353(53)$ & $0.99(0.91-1.08)$ & $1.00(0.91-1.09)$ \\
\hline
\end{tabular}


Table 4. Effect of the Mycobacterium tuberculosis Beijing lineage on the risk for infection by 12 months of follow-up in child and adult household contacts of culture-positive index patients, Lima, Peru, September 2009-August 2012

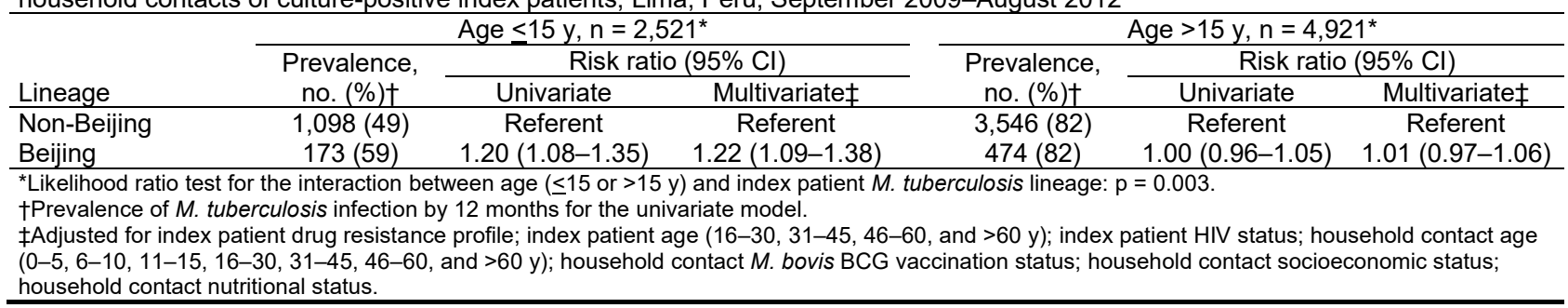

\section{TB at Baseline and by 12 Months of Follow-Up}

We used a modified Poisson generalized estimating equation to measure the association between exposure to index patients infected with Beijing strains and the likelihood of infection at baseline and by 12-months of follow-up. To account for correlation within households, we specified an exchangeable working correlation structure for observations within the same household and obtained empirical SE estimates for robust inference. We constructed univariate and multivariate models to compare the effect of exposure to Beijing and non-Beijing strains on the risk for TB infection at baseline and at 12 months. In the multivariate models, we included covariates from index patients (age, drug resistance profile, and HIV status) and their household contacts (age, diabetes status, BCG vaccination status, socioeconomic status, and nutritional status). We conducted sensitivity analyses in which we excluded household contacts who reported a history of TB, a previous positive TST result within 6 months after study enrollment, or a history of receiving TB treatment.

\section{Time to TST Conversion and to Incident Disease}

We assessed the time to TST conversion among household contacts who were uninfected at baseline by defining the date of infection as the midpoint between the date of enrollment and the date of a first positive TST result or TB diagnosis. We excluded from analysis household contacts who remained TST negative at the date of their last TST result. For the time to TB analysis, we excluded household contacts with co-prevalent TB and excluded from analysis household contacts in whom TB had not been diagnosed by the time of their death or at the end of the study. We first plotted Kaplan-Meier survival curves to visually evaluate the association between the index patient's $M$. tuberculosis genotype and the 2 time-to-event outcomes. We then used Cox frailty proportional hazards model to evaluate risk factors for time to TST conversion and time to incident disease while accounting for clustering within households. We constructed univariate and multivariate models to compare the effect of exposure to Beijing and non-Beijing strains on the risk for TST conversion and incident disease during follow-up. In the multivariate models, we included covariates from index patients (age, drug resistance profile, and HIV status) and their household contacts (age, diabetes status, BCG vaccination status, socioeconomic status, and nutritional status). We further adjusted for the use of IPT and TB history in the multivariate model of the time to incident disease analyses. We verified the proportional hazards assumptions for each covariate by including an interaction term for the covariate and time and stratifying by covariates for which the assumption did not hold. We conducted 2 sensitivity analyses, 1 in which we defined TST conversion using an increment of $\geq 6-\mathrm{mm}$ induration size in repeat TST measurements during follow-up and 1 in which we restricted the analysis to household contacts in whom active TB developed during follow-up and who received their diagnoses within $\geq 30$ days after index patient enrollment.

\section{Effect of Index Patient M. tuberculosis Lineage on Protective Efficacy of BCG Vaccination}

We also considered the possibility that index patient M. tuberculosis lineage might modify the protective

Table 5. Hazard ratios of tuberculin skin test conversion comparing contacts exposed to a Mycobacterium tuberculosis Beijing lineage with those exposed to a non-Beijing lineage, Lima, Peru, September 2009-August 2012

\begin{tabular}{|c|c|c|c|c|c|c|}
\hline \multirow[b]{3}{*}{ Lineage } & \multicolumn{3}{|c|}{ Age $\leq 15 \mathrm{y}^{*}$} & \multicolumn{3}{|c|}{ Age $>15 y^{*}$} \\
\hline & \multirow[b]{2}{*}{ Incidence, \%† } & \multicolumn{2}{|c|}{ Hazard ratio $(95 \% \mathrm{Cl})$} & \multirow[b]{2}{*}{ Incidence, \%† } & \multicolumn{2}{|c|}{ Hazard ratio $(95 \% \mathrm{Cl})$} \\
\hline & & Univariate & Multivariateł & & Univariate & Multivariate $\ddagger$ \\
\hline Non-Beijing & $0.26(356 / 1,396)$ & Referent & Referent & $0.69(845 / 1,236)$ & Referent & Referent \\
\hline Beijing & $0.39(62 / 160)$ & $1.53(1.09-2.14)$ & $1.65(1.17-2.33)$ & $0.73(118 / 162)$ & $1.02(0.80-1.30)$ & $1.03(0.80-1.33)$ \\
\hline
\end{tabular}


Table 6. Hazard ratios of incident tuberculosis among contacts exposed to a Mycobacterium tuberculosis Beijing lineage and a nonBeijing lineage, Lima, Peru, September 2009-August 2012

\begin{tabular}{|c|c|c|c|c|c|c|}
\hline \multirow[b]{3}{*}{ Lineage } & \multicolumn{3}{|c|}{ Age $\leq 15 \mathrm{y}^{*}$} & \multicolumn{3}{|c|}{ Age >15 $\mathrm{y}^{*}$} \\
\hline & \multirow[b]{2}{*}{ Incidence, \%† } & \multicolumn{2}{|c|}{ Hazard ratio $(95 \% \mathrm{Cl})$} & \multirow[b]{2}{*}{ Incidence, \%† } & \multicolumn{2}{|c|}{ Hazard ratio $(95 \% \mathrm{Cl})$} \\
\hline & & Univariate & Multivariate $\ddagger$ & & Univariate & Multivariate $\neq$ \\
\hline Non-Beijing & $3,043(84 / 2,760)$ & Referent & Referent & $2,874(156 / 4,993)$ & Referent & Referent \\
\hline Beijing & $4,478(15 / 335)$ & $1.42(0.78-2.59)$ & $1.45(0.77-2.72)$ & $3,124(19 / 661)$ & $0.93(0.57-1.52)$ & $1.06(0.64-1.77)$ \\
\hline \multicolumn{7}{|c|}{$\begin{array}{l}\text { *Likelihood ratio test for the interaction between age ( } \leq 15 \text { or }>15 \text { y) and index patient } M \text {. tuberculosis lineage: } p=0.231 \text {. } \\
\text { †Per } 100,000 \text { person-years in the univariate model. } \\
\text { †Adjusted for index patient drug resistance profile; index patient age }(16-30,31-45,46-60 \text {, and }>60 \text { y); index patient HIV status; household contact age } \\
(0-5,6-10,11-15,16-30,31-45,46-60 \text {, and }>60 \text { y); household contact } M \text {. bovis BCG vaccination status; household contact SES; household contact } \\
\text { nutritional status; household contact use of isoniazid preventive therapy; household contact TB history. }\end{array}$} \\
\hline
\end{tabular}

efficacy of BCG vaccine. To examine whether index patient $M$. tuberculosis strain type modified the association between BCG vaccination and outcomes of our study, we added in our model an interaction term that included index patient $M$. tuberculosis lineage and the BCG vaccination status of household contacts.

\section{Results}

We enrolled 9,151 household contacts of 2,223 culture-positive pulmonary TB index patients. The study enrollment rates were $85.5 \%$ for index patients and $94.6 \%$ for their household contacts. The retention rates for enrolled household contacts were $92.0 \%$ at 6 months and $94.7 \%$ at 12 months of follow-up. At baseline, $841(27.0 \%)$ of 3,115 child household contacts and 3,007 (53.1\%) of 5,663 adult household contacts were infected. A total of 951 (43\%) index patients had lineage 4.1 strain isolates, $775(35 \%)$ had lineage 4.3 strain isolates, $255(12 \%)$ had lineage 2 isolates (all of which were of the Beijing strain), and 242 (11\%) had other strain isolates (Table 1). We determined the baseline characteristics for household contacts, stratified by age at $\leq 15$ and $>15$ years (Table 2; Appendix Tables 1-4, https://wwwnc.cdc.gov/EID/ article/26/3/19-1314-App1.pdf).

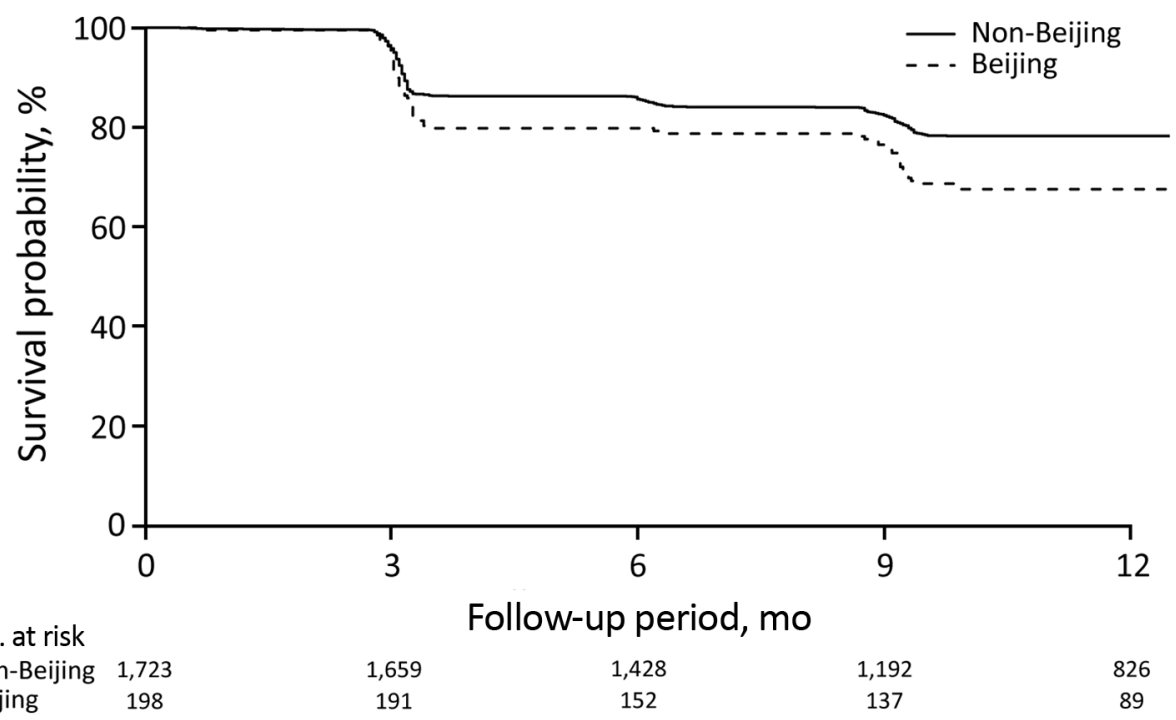

\section{M. tuberculosis Infection at Baseline and After 12 Months of Follow-up}

Child contacts exposed to Beijing strains were more likely than those exposed to non-Beijing strains to be infected at baseline (risk ratio [RR] 1.27 [95\% CI 1.061.53]; adjusted RR [aRR] 1.23 [95\% CI 1.02-1.50]) (Table 3 ) and by 12 months of follow-up (RR 1.20 [95\% CI 1.08-1.35]; aRR 1.22 [95\% CI 1.09-1.38]) (Table 4). The prevalence of $M$. tuberculosis infection at baseline (RR 0.99 [95\% CI 0.91-1.08]; aRR 1.00 [95\% CI 0.91-1.09]) and by 12 months (RR 1.00 [95\% CI 0.96-1.05]; aRR 1.01 [95\% CI 0.97-1.06]) did not vary by index patient M. tuberculosis strain in adult household contacts (Tables 3, 4). In the sensitivity analyses, we obtained almost identical results when we excluded household contacts who reported a history of $\mathrm{TB}$, a previous positive TST result within 6 months after study enrollment, or a history of TB treatment (Appendix Tables 5, 6).

\section{TST Conversion}

The TST status of children exposed to Beijing strains were more likely than that of children exposed to other strains to convert from negative to positive during 12 months of follow-up in both the univariate and

Figure 1. Survival curves for incident Mycobacterium tuberculosis infection in child household contacts by index patient $M$. tuberculosis lineage, Lima, Peru, September 2009August 2012. 


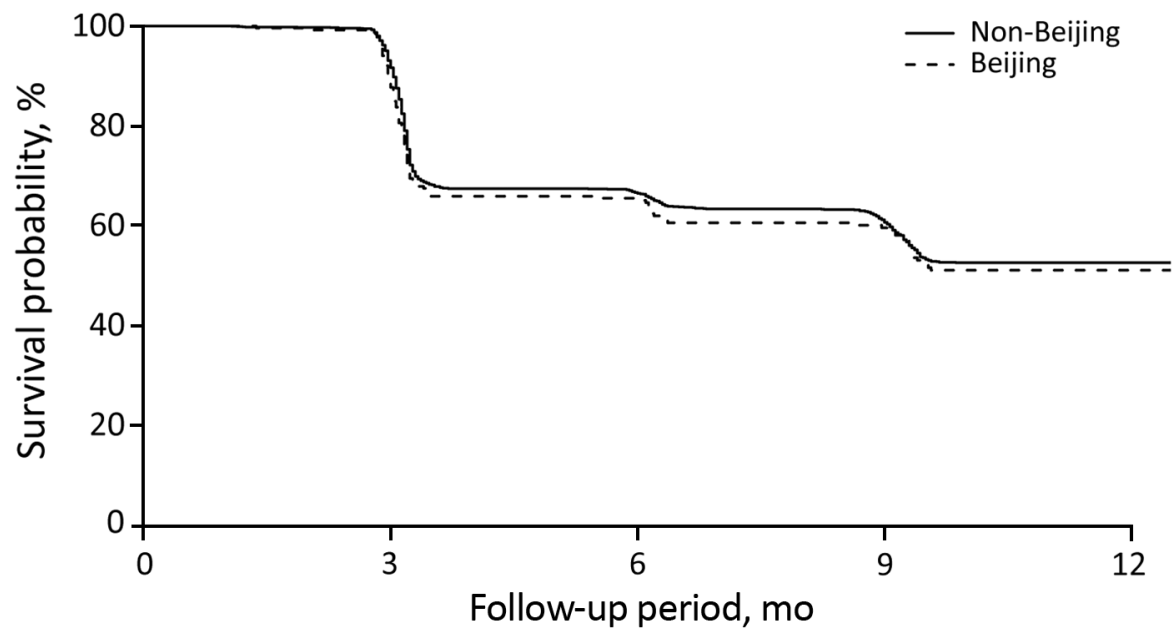

Figure 2. Survival curves for incident Mycobacterium tuberculosis infection in adult household contacts by index patient $M$. tuberculosis lineage, Lima, Peru, September 2009August 2012.

No. at risk

multivariate analyses (hazard ratio [HR] 1.53 [95\% CI 1.09-2.14]; adjusted HR [aHR] 1.65 [95\% CI 1.17-2.33]). Exposure to Beijing strains had no differential effect in adults (HR 1.02 [95\% CI 0.80-1.30]; aHR 1.03 [95\% CI 0.80-1.33]) (Table 5; Figures 1, 2). In the sensitivity analyses, we defined TST conversion by an increment of $\geq 6 \mathrm{~mm}$ in TST induration size during follow-up, and the results changed by $<5 \%$ (Appendix Table 7 ).

\section{Progression to Active TB}

After we adjusted for potential confounders, we found the HR for TB among child contacts exposed to an index patient with Beijing strains compared with nonBeijing strains to be 1.45 (95\% CI 0.77-2.7). We found no evidence that exposure to Beijing strains affected the risk for incident TB in adult contacts (aHR 1.06 [95\% CI 0.64-1.77]) (Table 6; Figures 3, 4). The results persisted when we restricted the analysis to household contacts in whom TB was diagnosed within $\geq 30$ days after index patient diagnosis (Appendix Table 8).

\section{Effect of Index Patient $M$. tuberculosis Lineage on the Protective Efficacy of BCG Vaccine}

Although they lacked statistical significance, the aRRs for TB outcomes were higher for BCG-vaccinated children exposed to Beijing strains than for non-BCGvaccinated children exposed to Beijing strains (aRR 1.01 [95\% CI 0.69-1.49] for M. tuberculosis infection at baseline; aRR 1.21 [95\% CI 0.90-1.63] for infection by 12 months; aRR 1.84 [95\% CI 0.77-4.39] for TST conversion; aRR 1.96 [95\% CI 0.41-9.39] for incident TB). This finding raises the possibility that BCG vaccination might be less efficacious in children exposed to Beijing strains than in those exposed to other strains

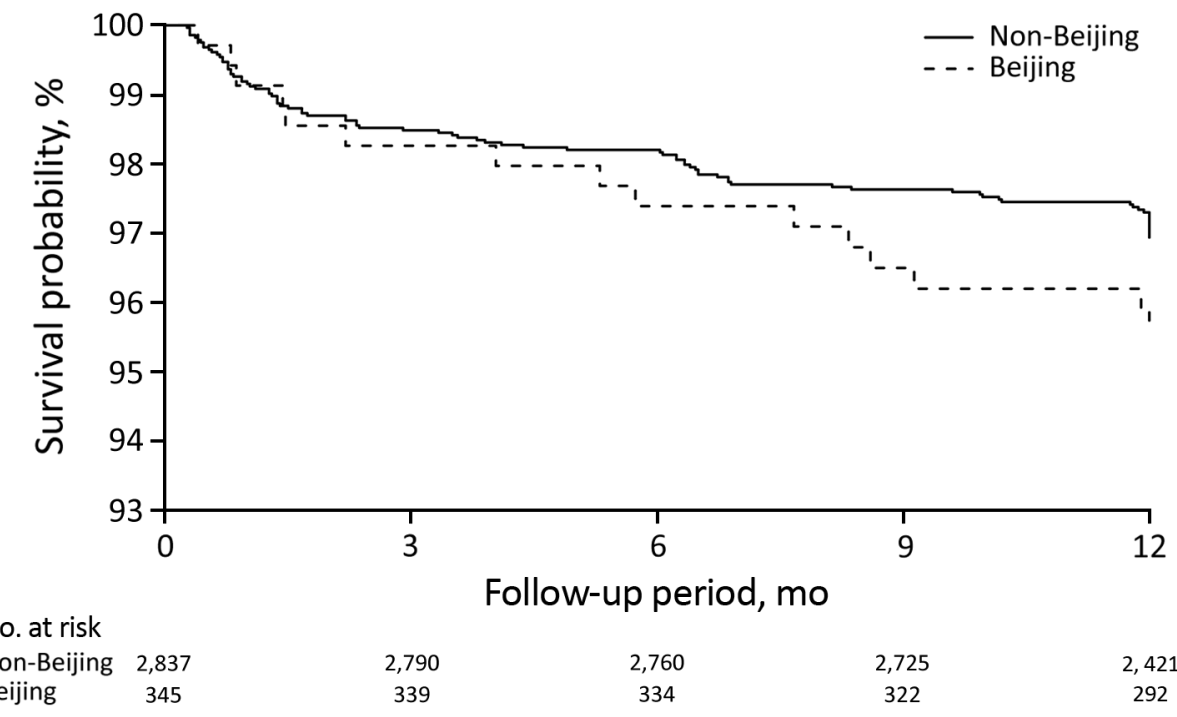

Figure 3. Survival curves for incident tuberculosis in child household contacts by index patient Mycobacterium tuberculosis lineage, Lima, Peru, September 2009-August 2012. 


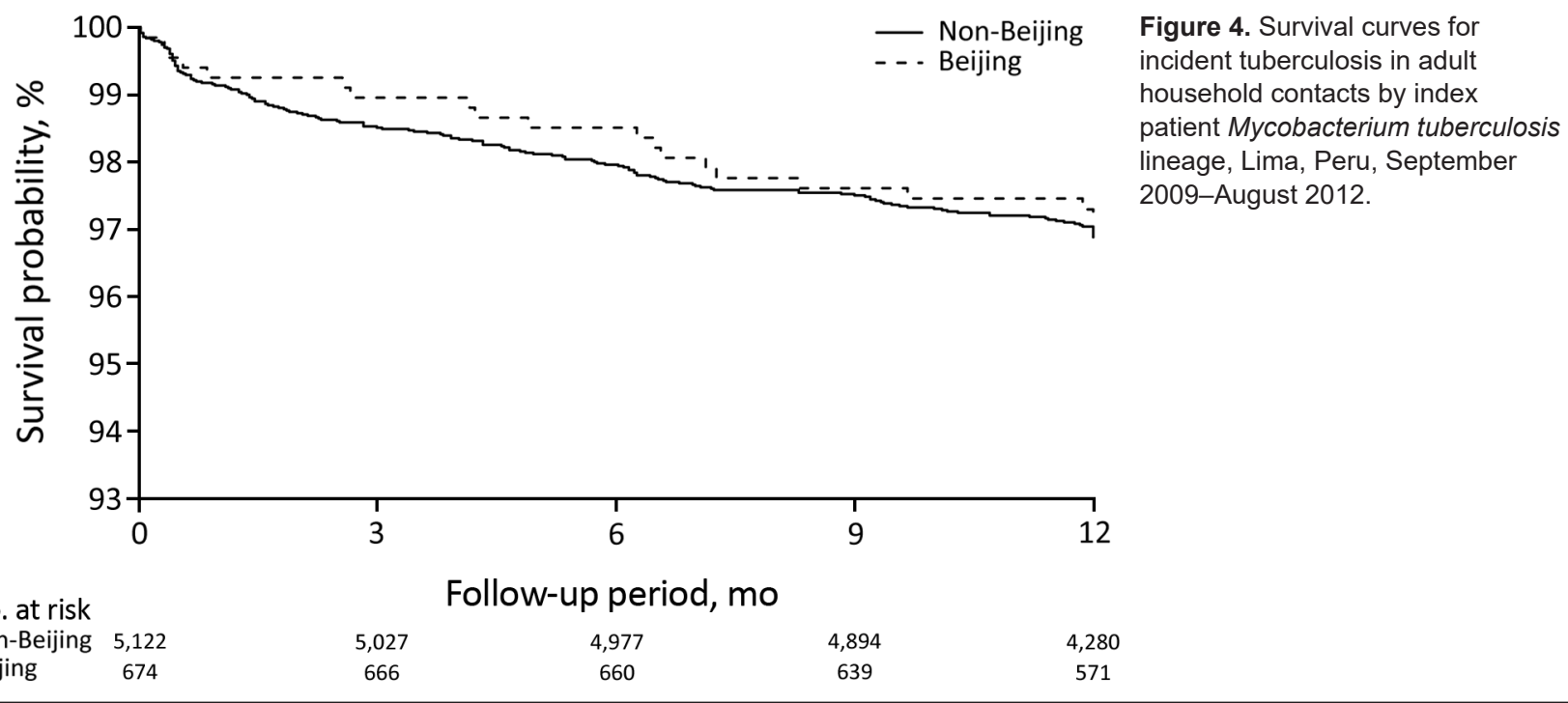

(Table 7). Among BCG-vaccinated adult household contacts exposed to Beijing strains, the aRRs were 1.00 (95\% CI 0.78-1.29) for M. tuberculosis infection at baseline, 1.02 (95\% CI 0.89-1.16) for infection by 12 months, 1.00 (95\% CI 0.48-2.06) for TST conversion, and 0.53 (95\% CI 0.17-1.68) for incident TB (Table 7).

\section{Discussion}

In our study, children exposed to strains of M. tuberculosis Beijing sublineage were more likely than were those exposed to strains of other lineages to become infected. In addition, children exposed to Beijing strains were more likely than children exposed to other strains to progress to $\mathrm{TB}$, although the relative risk of disease progression was not statistically significant (type 1 error rate of 0.05). We did not observe these effects among adult contacts.

Our results are consistent with those from previous studies that assessed the relative transmissibility of the Beijing strain through cohort-based studies or molecular epidemiology. In a study in South Africa, Marais et al. found that children $<5$ years of age who had household exposure to Beijing strains were 1.5 times more likely than those exposed to other strains to be TST-positive at baseline (13). In a prospective cohort study of household contacts in The Gambia, de Jong et al. found that Beijing lineage-exposed contacts were 7 times more likely than those exposed to the $M$. africanum lineage to progress to TB after 2 years of follow-up but were equally likely to be TST-positive at baseline or convert at 3 months (12). In the De Jong et al. study, the proportion of TST-positive household contacts at baseline and the incidence of TST conversion and disease did not differ significantly between Beijing and other strains within M. tuberculosis sensu strictu.
Although some molecular epidemiologic studies have found that Beijing strains are more likely than other strains to form genotypic clusters, other studies have not supported this conclusion. Many of these studies assumed that patients within a chain of recent $M$. tuberculosis transmission will share molecular fingerprints (32-36) and that clustering of genotypes is therefore a proxy for transmissibility and disease progression. In China, Yang et al. reported that patients infected with Beijing strains were 1.56 times more likely than those infected with other lineages to share molecular fingerprints with other patients (15). Similar findings have been reported from Lima; in a study by Imawoto et al., $80 \%$ of Beijing strains were in clusters, although clustering among other strains was not reported (14). In Vietnam, Holt et al. reported that, although the Beijing strains accounted for $58 \%$ of the total study sample, they constituted $70 \%$ of all clustered strains (21). In Papua New Guinea, Bainomugisa et al. found that $82 \%$ of isolates with Beijing strains were found in clusters, compared with $43 \%$ of isolates with strains from other lineages (37). In China, Yang et al. reported that the proportions of clustering were $34 \%$ for Beijing and $18 \%$ for non-Beijing strains (38). However, in Canada, Langlois-Klassen et al. found that Beijing was less likely to be found in clusters than other strains $(21 \%$ vs. $37 \%)(16)$, and in the Netherlands, Nebenzahl-Guimaraes et al. showed that Beijing strains were no more transmissible than strains of other lineages, after adjustment for host factors using an approach that controlled for the propensity of a strain to propagate (17).

Some animal studies have provided evidence to support the hypothesis that Beijing strains are more 
Table 7. Effects of BCG vaccination on $M$. tuberculosis infection and TB outcomes by lineage exposure for child and adult household contacts of culture-positive pulmonary tuberculosis patients, Lima, Peru, September 2009-August 2012*

\begin{tabular}{|c|c|c|c|c|c|}
\hline \multirow[b]{2}{*}{ Category and BCG vaccination status } & \multicolumn{2}{|c|}{ Non-Beijing lineage } & \multicolumn{2}{|c|}{ Beijing lineage } & \multirow[b]{2}{*}{ p value } \\
\hline & $\begin{array}{c}\text { Infection prevalence, } \\
\text { no. (\%) }\end{array}$ & $\mathrm{RR}(95 \% \mathrm{Cl})$ & $\begin{array}{c}\text { Infection prevalence, } \\
\text { no. }(\%)\end{array}$ & $\mathrm{RR}(95 \% \mathrm{Cl})$ & \\
\hline \multicolumn{6}{|l|}{ Children, $<15$ y } \\
\hline \multicolumn{5}{|c|}{ Baseline M. tuberculosis infection, $\mathrm{n}=2,949$} & \multirow[t]{3}{*}{0.691} \\
\hline Without BCG vaccination & $137(27)$ & Referent & $19(32)$ & Referent & \\
\hline With BCG vaccination & $552(26)$ & $0.94(0.81-1.09)$ & $83(32)$ & $1.01(0.69-1.49)$ & \\
\hline \multicolumn{5}{|c|}{ M. tuberculosis infection by 12 months of follow-up, $\mathrm{n}=2,417$} & \multirow[t]{4}{*}{0.405} \\
\hline Without BCG vaccination & $187(45)$ & Referent & $26(50)$ & Referent & \\
\hline \multirow[t]{2}{*}{ With $B C G$ vaccination } & $853(50)$ & $1.07(0.97-1.19)$ & $139(60)$ & $1.21(0.90-1.63)$ & \\
\hline & Incidence† & $\mathrm{HR}(95 \% \mathrm{Cl})$ & Incidence† & $\mathrm{HR}(95 \% \mathrm{Cl})$ & \\
\hline \multicolumn{5}{|l|}{ Time to TST conversion } & \multirow[t]{3}{*}{0.579} \\
\hline Without BCG vaccination & $0.18(48 / 260)$ & Referent & $0.21(7 / 32)$ & Referent & \\
\hline With BCG vaccination & $0.27(292 / 1,065)$ & $1.5(1.08-2.08)$ & $0.45(53 / 119)$ & $1.84(0.77-4.39)$ & \\
\hline Time to TB & & & & & \multirow[t]{4}{*}{0.168} \\
\hline Without BCG vaccination & $4,585(25 / 545)$ & Referent & $3,098(2 / 65)$ & Referent & \\
\hline \multirow[t]{2}{*}{ With $B C G$ vaccination } & $2,619(60 / 2291)$ & $0.58(0.35-0.96)$ & $4,639(13 / 280)$ & $1.96(0.41-9.39)$ & \\
\hline & $\begin{array}{c}\text { Infection prevalence, } \\
\text { no. }(\%)\end{array}$ & $\mathrm{RR}(95 \% \mathrm{Cl})$ & $\begin{array}{c}\text { Infection prevalence, } \\
\text { no. }(\%)\end{array}$ & $\operatorname{RR}(95 \% \mathrm{Cl})$ & \\
\hline \multicolumn{6}{|c|}{ Adults, $>15$ y } \\
\hline \multicolumn{5}{|c|}{ Baseline M. tuberculosis infection, $n=5,381$} & \multirow[t]{3}{*}{0.611} \\
\hline Without BCG vaccination & $260(47)$ & Referent & $27(51)$ & Referent & \\
\hline With BCG vaccination & $2,263(54)$ & $1.07(0.98-1.18)$ & $313(54)$ & $1.00(0.78-1.29)$ & \\
\hline \multicolumn{5}{|c|}{ M. tuberculosis infection by 12 months of follow-up, $n=4,716$} & \multirow[t]{4}{*}{0.315} \\
\hline Without BCG vaccination & $334(72)$ & Referent & $39(81)$ & Referent & \\
\hline \multirow[t]{2}{*}{ With $B C G$ vaccination } & $3,042(82)$ & $1.10(1.04-1.16)$ & $419(82)$ & $1.02(0.89-1.16)$ & \\
\hline & Incidence & $\mathrm{HR}(95 \% \mathrm{Cl})$ & Incidence† & $\mathrm{HR}(95 \% \mathrm{Cl})$ & \\
\hline \multicolumn{5}{|l|}{ Time to TST conversion } & \multirow[t]{3}{*}{0.315} \\
\hline Without BCG vaccination & $0.41(70 / 172)$ & Referent & $0.77(10 / 13)$ & Referent & \\
\hline With $B C G$ vaccination & $0.72(728 / 1,020)$ & $1.55(1.18-2.04)$ & $0.73(105 / 144)$ & $1.00(0.48-2.06)$ & \\
\hline Time to TB & & & & & \multirow[t]{3}{*}{0.732} \\
\hline Without BCG vaccination & $4,813(28 / 582)$ & Referent & $6,574(4 / 61)$ & Referent & \\
\hline With BCG vaccination & $2,860(129 / 4511)$ & $0.66(0.43-1.02)$ & $2,434(15 / 616)$ & $0.53(0.17-1.68)$ & \\
\hline
\end{tabular}

*BCG, M. bovis BCG; HR, hazard ratio; RR, risk ratio; TB, tuberculosis; TST, tuberculin skin test.

†Cases per person-year (no. converted/no. tested).

likely than other lineages to cause disease. Mice experimentally infected with Beijing strains not only died earlier and had higher death rates but also had more lung tissue damage than controls (39-42). Some in vitro studies of macrophages have also found that the Beijing strain can downregulate the expression of pathogen recognition receptors and major histocompatibility complex class II, modify the secretion of inflammatory cytokines, and induce the necrosis of host immune cells (43-46).

We considered possible explanations for the difference in the effect of the Beijing strain in children and adults. Given their mixing patterns, adults may be more likely than children to be infected within the community rather than in the household. If the household contacts in the cohort reported here had been infected by someone other than the household index patient, strain-specific exposure status might have been misclassified. Such misclassification would have been more likely in adults and would have biased the results for this group toward the null of no effect. However, another possibility is that Beijing strains might lead to earlier disease progression in younger persons with newly acquired $M$. tuberculosis. Several studies from Vietnam report that Beijing strains make up the highest proportion of incident cases in persons $<20$ years of age and that the prevalence of Beijing strains declines with increasing age $(18,19)$. In Iran, Erie et al. similarly showed that $27 \%$ of patients $\leq 20$ years of age were infected by Beijing strains and that the prevalence of Beijing strains among patients $\geq 20$ years of age was $13 \%(20)$. The increasing prevalence of Beijing strains in children may be related to use of BCG vaccine. This explanation would be consistent with our finding that that the protective efficacy of BCG vaccine against Beijing strains was reduced in children but not in adults. Possible explanations for these observations include a decrease in the protective efficacy of BCG vaccination with increasing age, a shift in the administered BCG strain in Peru's recent history, or a reduction in the immunogenicity of BCG vaccine over time (47-49).

Our study had several notable limitations. First, within a high-transmission setting such as Lima, children still could have been infected outside the household. Misclassification of the lineage exposure 
status in children also would have led to an underestimation of the effect. Second, discrepancies between 24-locus MIRU-VNTR genotyping and WGS have been noted previously (50), and some lineages assigned on the basis of MIRU-VNTR could have been inaccurate, again leading to a misclassification error that could have underestimated the true effect of Beijing strains.

In conclusion, we found that exposure to household index patients infected with Beijing strains was associated with increased risk for TST conversion and disease in children $\leq 15$ years of age but not in adults. These findings raise the possibility that genotypic variation in $M$. tuberculosis may have important phenotypic effects that should be further studied. In particular, it will be essential to determine whether BCG vaccination provides less protection against Beijing strains than strains from other lineages and whether the efficacy of any newly developed vaccines varies by $M$. tuberculosis genoype.

\section{Acknowledgments}

We thank the patients and their families who gave their time and energy to contribute to this study, the National Strategy for Tuberculosis Control at the Peruvian Ministry of Health, and the healthcare personnel at the 106 participating health centers in Lima.

This work was supported by the National Institute of Allergy and Infectious Diseases U19AI076217 and TBRU U19AI111224.

\section{About the Author}

Dr. Huang is an epidemiologist and biostatistician at Brigham and Women's Hospital and an instructor at Harvard Medical School. His primary research interest is in epidemiologic investigation of TB.

\section{References}

1. World Health Organization. Global tuberculosis report 2018. Geneva: The Organization; 2018.

2. Stucki D, Brites D, Jeljeli L, Coscolla M, Liu Q, Trauner A, et al. Mycobacterium tuberculosis lineage 4 comprises globally distributed and geographically restricted sublineages. Nat Genet. 2016;48:1535-43. https:/ / doi.org/10.1038/ng.3704

3. Bifani PJ, Mathema B, Kurepina NE, Kreiswirth BN. Global dissemination of the Mycobacterium tuberculosis W-Beijing family strains. Trends Microbiol. 2002;10:45-52. https:/ / doi.org/10.1016/S0966-842X(01)02277-6

4. Glynn JR, Whiteley J, Bifani PJ, Kremer K, van Soolingen D. Worldwide occurrence of Beijing/W strains of Mycobacterium tuberculosis: a systematic review. Emerg Infect Dis. 2002;8:843-9. https:// doi.org/10.3201/eid0805.020002

5. European Concerted Action on New Generation Genetic Markers and Techniques for the Epidemiology and Control of Tuberculosis. Beijing/W genotype Mycobacterium tuberculosis and drug resistance. Emerg Infect Dis. 2006;12:736-43. https:// doi.org/10.3201/eid1205.050400

6. Merker M, Blin C, Mona S, Duforet-Frebourg N, Lecher S, Willery E, et al. Evolutionary history and global spread of the Mycobacterium tuberculosis Beijing lineage. Nat Genet. 2015;47:242-9. https:// doi.org/10.1038/ng.3195

7. Agerton TB, Valway SE, Blinkhorn RJ, Shilkret KL, Reves R, Schluter WW, et al. Spread of strain W, a highly drug-resistant strain of Mycobacterium tuberculosis, across the United States. Clin Infect Dis. 1999;29:85-92, discussion 93-5. https:// doi.org/10.1086/520187

8. Caminero JA, Pena MJ, Campos-Herrero MI, Rodríguez JC, García I, Cabrera P, et al. Epidemiological evidence of the spread of a Mycobacterium tuberculosis strain of the Beijing genotype on Gran Canaria Island. Am J Respir Crit Care Med. 2001;164:1165-70. https://doi.org/10.1164/ ajrccm.164.7.2101031

9. Cowley D, Govender D, February B, Wolfe M, Steyn L, Evans J, et al. Recent and rapid emergence of W-Beijing strains of Mycobacterium tuberculosis in Cape Town, South Africa. Clin Infect Dis. 2008;47:1252-9. https:/ / doi.org/ $10.1086 / 592575$

10. Cox HS, Kubica T, Doshetov D, Kebede Y, Rüsch-Gerdess S, Niemann S. The Beijing genotype and drug resistant tuberculosis in the Aral Sea region of Central Asia. Respir Res. 2005;6:134. https://doi.org/10.1186/1465-9921-6-134

11. Hanekom M, van der Spuy GD, Streicher E, Ndabambi SL, McEvoy CRE, Kidd M, et al. A recently evolved sublineage of the Mycobacterium tuberculosis Beijing strain family is associated with an increased ability to spread and cause disease. J Clin Microbiol. 2007;45:1483-90. https:/ / doi.org/ 10.1128/JCM.02191-06

12. de Jong BC, Hill PC, Aiken A, Awine T, Antonio M, Adetifa IM, et al. Progression to active tuberculosis, but not transmission, varies by Mycobacterium tuberculosis lineage in The Gambia. J Infect Dis. 2008;198:1037-43. https:// doi.org/10.1086/591504

13. Marais BJ, Hesseling AC, Schaaf HS, Gie RP, van Helden PD, Warren RM. Mycobacterium tuberculosis transmission is not related to household genotype in a setting of high endemicity. J Clin Microbiol. 2009;47:1338-43. https:/ / doi.org/10.1128/JCM.02490-08

14. Iwamoto T, Grandjean L, Arikawa K, Nakanishi N, Caviedes L, Coronel J, et al. Genetic diversity and transmission characteristics of Beijing family strains of Mycobacterium tuberculosis in Peru. PLoS One. 2012;7:e49651. https://doi.org/10.1371/journal.pone.0049651

15. Yang C, Shen X, Peng Y, Lan R, Zhao Y, Long B, et al. Transmission of Mycobacterium tuberculosis in China: a population-based molecular epidemiologic study. Clin Infect Dis. 2015;61:219-27. https://doi.org/10.1093/cid/civ255

16. Langlois-Klassen D, Senthilselvan A, Chui L, Kunimoto D, Saunders LD, Menzies D, et al. Transmission of Mycobacterium tuberculosis Beijing strains, Alberta, Canada, 1991-2007. Emerg Infect Dis. 2013;19:701-11. https://doi.org/ 10.3201/eid1905.121578

17. Nebenzahl-Guimaraes H, Borgdorff MW, Murray MB, van Soolingen D. A novel approach - the propensity to propagate (PTP) method for controlling for host factors in studying the transmission of Mycobacterium tuberculosis. PLoS One. 2014;9:e97816. https:/ / doi.org/10.1371/ journal.pone.0097816

18. Anh DD, Borgdorff MW, Van LN, Lan NT, van Gorkom T, Kremer K, et al. Mycobacterium tuberculosis Beijing $g$ enotype emerging in Vietnam. Emerg Infect Dis. 2000; 6:302-5. https://doi.org/10.3201/eid0603.000312 
19. Buu TN, Huyen MN, Lan NTN, Quy HT, Hen NV, Zignol M, et al. The Beijing genotype is associated with young age and multidrug-resistant tuberculosis in rural Vietnam. Int J Tuberc Lung Dis. 2009;13:900-6.

20. Erie H, Kaboosi H, Javid N, Shirzad-Aski H, Taziki M, Kuchaksaraee MB, et al. The high prevalence of Mycobacterium tuberculosis Beijing strain at an early age and extra-pulmonary tuberculosis cases. Iran J Microbiol. 2017;9:312-7.

21. Holt KE, McAdam P, Thai PVK, Thuong NTT, Ha DTM, Lan NN, et al. Frequent transmission of the Mycobacterium tuberculosis Beijing lineage and positive selection for the EsxW Beijing variant in Vietnam. Nat Genet. 2018;50:849-56. https://doi.org/10.1038/s41588-018-0117-9

22. Becerra MC, Huang CC, Lecca L, Bayona J, Contreras C, Calderon R, et al. Transmissibility and potential for disease progression of drug resistant Mycobacterium tuberculosis: prospective cohort study. BMJ. 2019;367:15894. https:/ / doi.org/10.1136/bmj.15894

23. Ministerio de Salud. Norma técnica de salud para el control de la tuberculosis. Lima, Peru. 2006 [cited 2018 Oct 10]. $\mathrm{ftp}: / / \mathrm{ftp} 2 . \mathrm{minsa}$.gob.pe/descargas/dgsp/ESNtuberculosis/normaspublicaciones/NTSTBC.pdf

23. Joshi N, Fass J. A sliding-window, adaptive, quality-based trimming tool for FastQ files. 2011 [cited 2018 Dec 15]. https://github.com/najoshi/sickle

24. Li H. Toward better understanding of artifacts in variant calling from high-coverage samples. Bioinformatics. 2014;30:2843-51. https://doi.org/10.1093/bioinformatics/ btu356

25. Li H, Handsaker B, Wysoker A, Fennell T, Ruan J, Homer N, et al.; 1000 Genome Project Data Processing Subgroup. The Sequence Alignment/Map format and SAMtools. Bioinformatics. 2009;25:2078-9. https:// doi.org/10.1093/ bioinformatics/btp352

26. Walker BJ, Abeel T, Shea T, Priest M, Abouelliel A, Sakthikumar S, et al. Pilon: an integrated tool for comprehensive microbial variant detection and genome assembly improvement. PLoS One. 2014;9:e112963. https:/ / doi.org/ 10.1371/journal.pone.0112963

27. Coll F, McNerney R, Guerra-Assunção JA, Glynn JR, Perdigão J, Viveiros M, et al. A robust SNP barcode for typing Mycobacterium tuberculosis complex strains. Nat Commun. 2014;5:4812. https://doi.org/10.1038/ ncomms 5812

28. Supply P, Lesjean S, Savine E, Kremer K, van Soolingen D, Locht $\mathrm{C}$. Automated high-throughput genotyping for study of global epidemiology of Mycobacterium tuberculosis based on mycobacterial interspersed repetitive units. J Clin Microbiol. 2001;39:3563-71. https:/ / doi.org/10.1128/ JCM.39.10.3563-3571.2001

29. Allix-Béguec C, Harmsen D, Weniger T, Supply P, Niemann S. Evaluation and strategy for use of MIRUVNTRplus, a multifunctional database for online analysis of genotyping data and phylogenetic identification of Mycobacterium tuberculosis complex isolates. J Clin Microbiol. 2008;46:2692-9. https:// doi.org/10.1128/JCM.00540-08

30. Verrall AJ, Netea MG, Alisjahbana B, Hill PC, van Crevel R. Early clearance of Mycobacterium tuberculosis: a new frontier in prevention. Immunology. 2014;141:506-13. https:/ / doi.org/10.1111/imm.12223

31. Graham SM, Ahmed T, Amanullah F, Browning R, Cardenas V, Casenghi M, et al. Evaluation of tuberculosis diagnostics in children: 1 . Proposed clinical case definitions for classification of intrathoracic tuberculosis disease. Consensus from an expert panel. J Infect Dis.
2012;205(Suppl 2):S199-208. https:/ / doi.org/10.1093/ infdis/jis008

32. Toungoussova OS, Mariandyshev A, Bjune G, Sandven P, Caugant DA. Molecular epidemiology and drug resistance of Mycobacterium tuberculosis isolates in the Archangel prison in Russia: predominance of the W-Beijing clone family. Clin Infect Dis. 2003;37:665-72. https:/ / doi.org/ 10.1086/377205

33. Dou H-Y, Tseng F-C, Lin C-W, Chang J-R, Sun J-R, Tsai W-S, et al. Molecular epidemiology and evolutionary genetics of Mycobacterium tuberculosis in Taipei. BMC Infect Dis. 2008;8:170. https:// doi.org/10.1186/1471-2334-8-170

34. Wada T, Fujihara S, Shimouchi A, Harada M, Ogura H, Matsumoto S, et al. High transmissibility of the modern Beijing Mycobacterium tuberculosis in homeless patients of Japan. Tuberculosis (Edinb). 2009;89:252-5. https:/ / doi.org/ 10.1016/j.tube.2009.05.007

35. Yang C, Luo T, Sun G, Qiao K, Sun G, DeRiemer K, et al. Mycobacterium tuberculosis Beijing strains favor transmission but not drug resistance in China. Clin Infect Dis. 2012;55:1179-87. https://doi.org/10.1093/cid/cis670

36. Aleksic E, Merker M, Cox H, Reiher B, Sekawi Z, Hearps AC, et al. First molecular epidemiology study of Mycobacterium tuberculosis in Kiribati. PLoS One. 2013;8:e55423. https://doi.org/10.1371/journal.pone.0055423

37. Bainomugisa A, Pandey S, Donnan E, Simpson G, Foster J, Lavu E, et al. Cross-border movement of highly drug-resistant Mycobacterium tuberculosis from Papua New Guinea to Australia through Torres Strait Protected Zone, 2010-2015. Emerg Infect Dis. 2019;25:406-15. https://doi.org/10.3201/eid2503.181003

38. Yang C, Luo T, Shen X, Wu J, Gan M, Xu P, et al. Transmission of multidrug-resistant Mycobacterium tuberculosis in Shanghai, China: a retrospective observational study using whole-genome sequencing and epidemiological investigation. Lancet Infect Dis. 2017;17:275-84. https:// doi.org/10.1016/S1473-3099(16)30418-2

39. Manca C, Tsenova L, Bergtold A, Freeman S, Tovey M, Musser JM, et al. Virulence of a Mycobacterium tuberculosis clinical isolate in mice is determined by failure to induce Th1 type immunity and is associated with induction of IFN- $\alpha / \beta$. Proc Natl Acad Sci U S A. 2001;98:5752-7. https:// doi.org/10.1073/pnas.091096998

40. López B, Aguilar D, Orozco H, Burger M, Espitia C, Ritacco V, et al. A marked difference in pathogenesis and immune response induced by different Mycobacterium tuberculosis genotypes. Clin Exp Immunol. 2003;133:30-7. https://doi.org/10.1046/j.1365-2249.2003.02171.x

41. Dormans J, Burger M, Aguilar D, Hernandez-Pando R, Kremer K, Roholl P, et al. Correlation of virulence, lung pathology, bacterial load and delayed type hypersensitivity responses after infection with different Mycobacterium tuberculosis genotypes in a BALB/c mouse model. Clin Exp Immunol. 2004;137:460-8. https:/ / doi.org/10.1111/ j.1365-2249.2004.02551.x

42. Aguilar D, Hanekom M, Mata D, Gey van Pittius NC, van Helden PD, Warren RM, et al. Mycobacterium tuberculosis strains with the Beijing genotype demonstrate variability in virulence associated with transmission. Tuberculosis (Edinb). 2010;90:319-25. https:// doi.org/10.1016/j.tube.2010.08.004

43. Rocha-Ramírez LM, Estrada-García I, López-Marín LM, Segura-Salinas E, Méndez-Aragón P, Van Soolingen D, et al. Mycobacterium tuberculosis lipids regulate cytokines, TLR-2/4 and MHC class II expression in human macrophages. Tuberculosis (Edinb). 2008;88:212-20. https://doi.org/ 10.1016/j.tube.2007.10.003 
44. Chacón-Salinas R, Serafín-López J, Ramos-Payán R, Méndez-Aragón P, Hernández-Pando R, Van Soolingen D, et al. Differential pattern of cytokine expression by macrophages infected in vitro with different Mycobacterium tuberculosis genotypes. Clin Exp Immunol. 2005;140:443-9. https://doi.org/10.1111/j.1365-2249. 2005.02797.x

45. Sohn H, Lee K-S, Kim S-Y, Shin D-M, Shin S-J, Jo E-K, et al. Induction of cell death in human macrophages by a highly virulent Korean Isolate of Mycobacterium tuberculosis and the virulent strain H37Rv. Scand J Immunol. 2009;69:43-50. https://doi.org/10.1111/j.1365-3083.2008.02188.x

46. van Laarhoven A, Mandemakers JJ, Kleinnijenhuis J, Enaimi M, Lachmandas E, Joosten LAB, et al. Low induction of proinflammatory cytokines parallels evolutionary success of modern strains within the Mycobacterium tuberculosis Beijing genotype. Infect Immun. 2013;81:3750-6. https://doi.org/10.1128/IAI.00282-13
47. Sterne JA, Rodrigues LC, Guedes IN. Does the efficacy of BCG decline with time since vaccination? Int J Tuberc Lung Dis. 1998;2:200-7.

48. Luca S, Mihaescu T. History of BCG vaccine. Maedica (Buchar). 2013;8:53-8.

49. Behr MA. BCG - different strains, different vaccines? Lancet Infect Dis. 2002;2:86-92. https://doi.org/10.1016/ S1473-3099(02)00182-2

50. Roetzer A, Diel R, Kohl TA, Rückert C, Nübel U, Blom J, et al. Whole genome sequencing versus traditional genotyping for investigation of a Mycobacterium tuberculosis outbreak: a longitudinal molecular epidemiological study. PLoS Med. 2013; 10:e1001387. https:/ / doi.org/10.1371/journal.pmed.1001387

Address for correspondence: Megan B. Murray, Harvard Medical School, 641 Huntington Ave, Boston, MA 02115, USA; email: megan.murray.epi@gmail.com

\section{Emerging Infectious Diseases Spotight Topics}
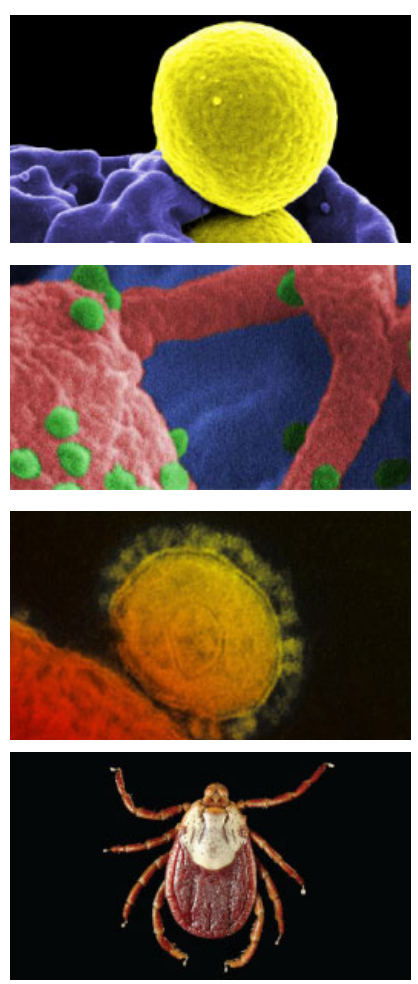
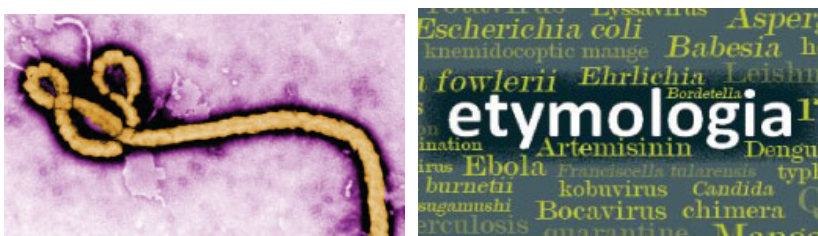

Antimicrobial resistance • Ebola Etymologia Food safety • HIV-AIDS Influenza • Lyme disease • Malaria MERS • Pneumonia • Rabies Tuberculosis • Ticks • Zika
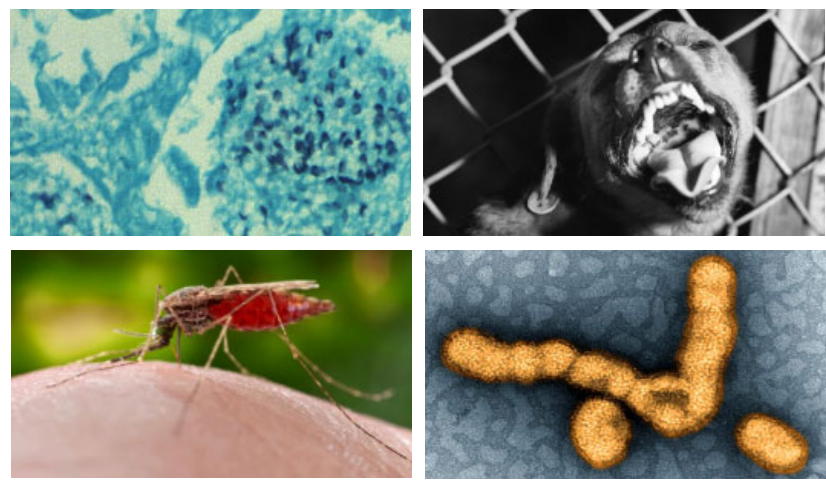
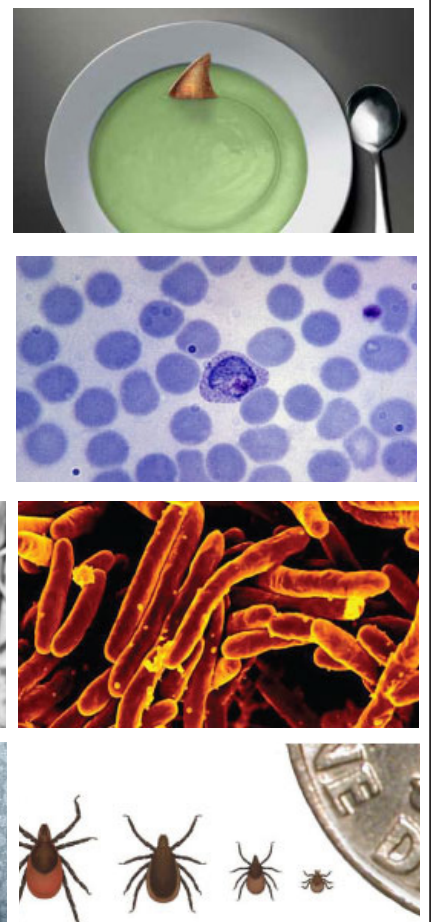

EID's spotlight topics highlight the latest articles and information on emerging infectious disease topics in our global community

https://wwwnc.cdc.gov/eid/page/spotlight-topics 